\title{
Pollos de engorde alimentados con dietas de consumo restringido que contienen aceite de palma de seje (Oenocarpus bataua)
}

Broilers fed with diets of restricted consumption containing seje palm oil

(Oenocarpus bataua)

\author{
Fernández Lavado Andrea Paola y Ocampo Duran Álvaro ${ }^{2}$ \\ ${ }^{1} \mathrm{MVZ}$, Unillanos y ${ }^{2}$ Zoot, MSc, PhD \\ Grupo de Investigación Sistemas Sostenibles de Producción con énfasis en \\ Palmas Tropicales Universidad de los Llanos \\ aocampo@unillanos.edu.co
}

Recibido 12 de Diciembre 2013, Aceptado 26 de Septiembre 2014

\section{RESUMEN}

Para que la industria avícola a pequeña escala sea rentable, es necesario encontrar alternativas de alimentación, utilizando materias primas disponibles en cada región, es así, que la palma de seje (Oenocarpus bataua) es un recurso nativo, valioso y con un bajo uso en la región Orinoquia; de su fruto se obtiene un aceite de alta calidad nutricional, similar en su contenido de ácidos grasos al del aceite de Oliva. Este experimento se realizó en la Reserva Natural Las Unamas, ubicada en el municipio de San Martín, Meta, se utilizaron setenta y dos pollos de engorde, en un diseño completamente al azar con cuatro tratamientos y tres repeticiones de seis pollos cada una, a los que se les suministraron dietas con consumo restringido con diferentes niveles de aceite de palma de seje, los cuatro tratamientos valorados fueron: T1 = concentrado comercial (energía a partir de carbohidratos), T2 = fuente energética el aceite de palma africana (APA), T3 = reemplazo del $10 \%$ del APA por aceite de seje (AS), y T4 = reemplazo del $20 \%$ del APA por AS. Las variables evaluadas fueron la ganancia de peso total $(\mathrm{g})$ : 1362 , 1179, 1530.8 y 1678.6 para el T1, T2, T3 y T4 respectivamente $(\mathrm{P}<0.082)$, lo cual indica que los tratamientos fueron similares; el rendimiento en canal fue 66.7, 58.2, 61.9 y $63.2 \%$ respectivamente $(P<0.01)$, indicando que los aceites en las dietas de los pollos de engorde afectaron negativamente su rendimiento de la canal. La grasa abdominal mostró una mayor consistencia y color amarillo en T1, siendo 
esta de menor calidad, en comparación con la de color blanco y de baja consistencia en T2, T3 y T4. En la prueba de degustación, la carne más apetecida (43.3\%) fue la del T3 (90\% APA y $10 \%$ AS), seguida del T4 (80\% APA y $20 \%$ AS), T2 (100\% APA) y finalmente T1 (dieta comercial-carbohidratos). Se concluye que es viable el uso de dietas ricas en ácidos grasos para la alimentación de pollos de engorde, puesto que la inclusión del total de las fuentes de aceite del 10 y $20 \%$ de AS, influyó positivamente la composición final de ácidos grasos de su carne, favoreciendo su consistencia y palatabilidad.

Palabras clave: Palma africana, ácidos grasos, alimentación, energía.

\section{ABSTRACT}

For the small-scale poultry industry be profitable, is necessary to find alternative feeding, using raw materials available in each region, so that palm of seje (Oenocarpus bataua) is a native resource, valuable and low use in the Orinoco region; its fruit oil of high nutritional quality is obtained, similar in content to the fatty acids of olive oil. This experiment was conducted in the Natural Reserve Las Unamas, located in the municipality of San Martin, Meta, seventy two broilers were used, in a completely randomized design with four treatments and three replicates of six chicks each one, to which are supplied with restricted diets with different levels of consumption of oil seje palm, the four treatments were evaluated: $\mathrm{T} 1=$ commercial concentrate (energy from carbohydrates), T2 = energy source palm oil (APA), T3 $=10 \%$ replacement of oil by APA seje (AS), T3 $=$ replacement of $10 \%$ APA by oil seje (AS), and T4 = replacement of $20 \%$ APA by AS. The evaluated variables were the total weight gain $(\mathrm{g}): 1362,1179,1530.8$ and 1678.6 for T1, T2, T3 and T4 respectively $(P<0.082)$, indicating that the treatments were similar; performance channel was 66.7, 58.2, 61.9 and 63.2\% respectively $(P<0.01)$, indicating that the oils in the diets of broilers negatively affected their performance channel. Abdominal fat showed more consistency and yellow color in T1, this being lower quality, compared to white color and low consistent in T2, T3 and T4. In the taste test, the most desired meat (43.3\%) was T3 (90\% APA and 10\% AS), followed by T4 ( $80 \%$ APA and $20 \%$ AS), T2 (100\% APA) and finally T1 
(commercial diet - carbohydrates). We conclude that it is feasible the use of diets rich in fatty acids to feed broilers, since the inclusion of all oil sources 10 and $20 \%$ AS, positively influences the final composition of fatty acid of his meat favoring his consistency and palatability.

Keywords: African palm, fatty acids, food, energy.

\section{RESUMO}

Para a pequena escala indústria avícola é rentável, você precisa encontrar fornecimentos alternativos, utilizando matérias-primas disponíveis em cada região, de modo que a palma da seje (patauá) é um recurso natural, uso valioso e com baixo custo na região do Orinoco; o seu fruto se obtem um óleo de alta qualidade nutricional, similar em conteúdo para os ácidos graxos de óleo de oliva. Este experimento foi realizado na Reserva Natural Las Unamas, localizado no município de San Martin, Meta, foram utilizados setenta e dois frangos de corte, em um delineamento inteiramente casualizado, com quatro tratamentos e três repetições de seis aves cada um, a que são fornecidos com dietas restritas com diferentes níveis de consumo de óleo de palma de seje, os quatro tratamentos avaliados foram: $\mathrm{T} 1$ = concentrado comercial (energia a partir de carboidratos), T2 = óleo de palma africano fonte energia (APA), T3 = substituição do 10\% ० APA por óleo de seje (AS), e T4 = substituição de $20 \%$ ○ APA por AS. As variáveis estudadas foram o ganho de peso total $(\mathrm{g})$ : 1362, 1179, 1530.8 e 1678.6 de T1, T2, T3 e T4, respectivamente $(\mathrm{P}<0.082)$, indicando que os tratamentos foram similares; rendimento de carcaça foi de 66.7, 58.2, 61.9 e 63.2\%, respectivamente $(P<0.01)$, indicando que os óleos nas dietas de frangos de corte afetou negativamente o desempenho de seu canal. A gordura abdominal mostrou mais consistência e cor amarelo em $\mathrm{T} 1$, sendo este de qualidade inferior, em comparação com o cor branco e de baixa consistência em T2, T3 e T4. No teste de sabor, a carne mais desejado (43.3\%) era T3 (90\% APA e $10 \%$ AS), seguido de T4 ( $80 \%$ de APA e $20 \%$ de AS), T2 (100\% APA) e, finalmente, T1 (dieta comercial - hidratos de carbono). Concluímos que é viável a utilização de dietas ricas em ácidos graxos para alimentar frangos de corte, uma vez que a inclusão 
de todas as fontes de óleo o 10 e $20 \%$ de AS, influencia positivamente a composição final de ácidos gordos da sua carne favorecendo sua consistência e palatabilidade.

Palavras-chave: Palma africana, ácidos graxos, alimentos, energia.

\section{INTRODUCCIÓN}

Tradicionalmente se utiliza como fuente energética en los programas de nutrición para la producción animal, los carbohidratos que son aportados por los cereales, lo cual es considerado, muy competitivo basado en la demanda generada por la alimentación humana (Ordoñez, 2006).

Como alternativas de fuentes energéticas para la alimentación animal, se cuenta con los aceites vegetales que son producidos por las palmas, de las cuales, se han reportado aproximadamente 200 géneros y 1.500 especies, que particularmente se encuentran en el trópico y subtrópico 67 géneros y 550 especies se encuentran en América (Borchsenius y Moraes, 2006). Esto indica que las palmas son uno de los recursos más biodiversos con que cuenta el hombre. En la Orinoquia Colombiana existen alrededor de 46 especies que son la base de los pobladores, en lo que se refiere a su seguridad alimentaria (Correa et al., 2005; Banco de Occidente, 2005).

La palma de seje (Oenocarpus bataua) es un recurso nativo, valioso y con un bajo uso en el departamento del Meta y la región Orinoquía, de su fruto se obtiene un aceite de alta calidad nutricional con elevado contenido de ácidos grasos insaturados similares a los ofrecidos por el aceite de Oliva. Igualmente del proceso de extracción, se obtienen subproductos con un alto nivel proteico y mineral (Ocampo et al., 2013). La palma de seje (Oenocarpus bataua) es poco conocida, aunque existe información parcial de las características generales del aceite, es necesario precisar aspectos relacionados con el potencial de esta palma que es un cultivo natural con condiciones orgánicas de producción (Alfonso, 2009). Para establecer las ventajas del aceite de seje en la alimentación animal, el objetivo de este trabajo fue utilizar esta palma para enriquecer dietas ofrecidas a pollos de 
engorde y determinar la ganancia de peso y su rendimiento en canal, calidad de la grasa y carne.

\section{METODOLOGÍA}

El trabajo de campo se llevó a cabo en la Reserva Natural Las Unamas, adscrita a la Red Colombiana de reservas Privadas de la Sociedad civil, Nodo Orinoquia coordinado por la Fundación Horizonte Verde en el departamento del Meta, se encuentra ubicada en el municipio de San Martín, Meta, hacienda las Pampas a $30 \mathrm{~km}$ del casco urbano, vereda La Novilla, donde existe un cultivo de palma de seje de aproximadamente 300 hectáreas con palmas en producción. La reserva se encuentra a una altura de $300 \mathrm{msnm}$, su clima es de cálido húmedo a muy húmedo tropical; su humedad relativa es alta en toda el área y alcanza valores de $85 \%$ en la estación húmeda y del 50 al $60 \%$ en la estación seca, el promedio de temperatura es de $27^{\circ} \mathrm{C}$ pero en época de verano puede alcanzar los $34^{\circ} \mathrm{C}$ (IDEAM, 2014).

Por la reserva circulan los caños Camoa y Cumaral y atravesándola el caño Chumeco, el paisaje predominante está definido por sabanas bien drenadas onduladas, matizadas por bosques de galería y bosques en crecimiento. La vegetación es abundante y variada, con elementos amazónicos, la cual tiene un alto grado de conservación, y la regeneración de la misma se hace en forma natural con bosques secundarios.

\section{Dietas utilizadas para pollos de engorde}

Como este experimento con pollos de engorde se efectuó en época de verano (Diciembre a Febrero), en donde la temperatura promedio en la Reserva Natural Las Unamas llega a $34^{\circ} \mathrm{C}$ bajo sombra, se realizaron las respectivas adecuaciones de las instalaciones para el bienestar de las aves.

Para la realización del estudio se utilizaron como animales experimentales 72 pollos de engorde de la raza Cobb, los cuales fueron alimentados durante 15 días con alimento balanceado de pre-inicio, siendo su contenido de proteína $22 \%$, 
grasa $3 \%$, fibra $5 \%$, cenizas $8 \%$ y humedad $3 \%$, el alimento se suministró a voluntad y el agua fue tratada con cloro ( $1 \mathrm{ml} / 25 \mathrm{~L}$ de agua), tomando como referencia trabajos anteriores realizados por Herrera y Quintero, (1996).

Los bebederos y comederos se lavaron y desinfectaron con yodo en una fracción de $1 \mathrm{ml}$ para $10 \mathrm{~L}$ de agua cada tercer día. Los bebederos se llenaron tres veces al día para proporcionar agua fresca y así ayudar a refrescar las aves cuando la temperatura en la instalación era alta. El experimento se inició con 80 pollitos, de los cuales se murieron dos en el periodo de preinicio, lo que equivale a un $2.5 \%$ de mortalidad, sacando seis pollitos para que las tres repeticiones de cada tratamiento quedaran con igual número de unidades experimentales.

En la semana tres se pesaron las aves y se dividió el número total de aves en los cuatro tratamientos, distribuidas en un diseño completamente al azar, con tres repeticiones cada una y seis unidades experimentales, utilizando un área de 0.7 $\mathrm{m}^{2}$ por ave, con una cama de ocho $\mathrm{cm}$ de cascarilla de arroz. En esta semana se inició el acostumbramiento a las diferentes dietas, los aceites de palma y seje, se fueron sustituyendo así: $10 \%$ el primer día, $25 \%$ el segundo, $40 \%$ el tercer y cuarto, $65 \%$ el quinto, $80 \%$ el sexto y séptimo, y luego $100 \%$ en el octavo día, esto con el fin de evitar cambios bruscos en la dieta que consumían las aves (Tablas 1 y 2). El modelo estadístico fue el siguiente:

$$
Y i j k=\mu+T i+\varepsilon i j k
$$

En donde, $\boldsymbol{Y} \boldsymbol{i} \boldsymbol{j} \boldsymbol{k}=$ variable respuesta de la puntuación del $\boldsymbol{i}$ sujeto sometido al $\boldsymbol{j}$ tratamiento, $\boldsymbol{\mu}=$ efecto de la media poblacional, $\boldsymbol{T} \boldsymbol{i}=$ efecto del tratamiento $\boldsymbol{i}$ en las variables de: aumento de peso, rendimiento en canal, eficiencia alimenticia y calidad de carne y grasa, $\boldsymbol{\varepsilon} \boldsymbol{i} \boldsymbol{j} \boldsymbol{k}=$ error experimental $\mathrm{o}$ efecto aleatorio del muestreo.

Las instalaciones donde se formaron los corrales no eran las ideales para la explotación avícola, debido a una deficiencia en la ventilación, una altura de los muros externos de $1.20 \mathrm{~m}$ y la del techo de $2.35 \mathrm{~m}$, lo cual impedía la circulación del aire y permitía la concentración de la temperatura dentro del galpón. Para 
evitar la concentración de amoniaco y el aumento de la temperatura de la cama, se realizó dos veces al día volteo de la cascarilla lo cual ayudó a disminuir la humedad dejada por el consumo de agua. Las aves duraron un periodo de 45 días consumiendo la dieta designada para cada tratamiento, donde se pesaron semanalmente todas las aves de cada tratamiento.

En el tratamiento 1 (T1) o control se empleó concentrado suministrado diariamente en la cantidad recomendada para la raza Cobb (promedio de $112.71 \mathrm{~g} /$ día, total $5702.1 \mathrm{~g} / 45$ días) (Cobb-vantresss, 2013); en los tratamientos dos (T2), tres (T3) y cuatro (T4) se empleó torta de soya enriquecida con vitaminas y minerales como fuente de proteína y suministrando la energía los ácidos grasos provenientes del aceite crudo de palma y seje, además se incluyeron los carbohidratos representados por la harina de arroz (Tablas 1 y 2). A los animales sometidos a estos tratamientos que fueron isoenergeticos e isoproteicos solo se les suministró el $70 \%$ de la ración diaria recomendada para la raza Cobb (promedio de 97.84 g/día) para evitar el exceso de grasa en los tejidos, en base a ensayos anteriores realizados por Herrera y Quintero, (1996).

Tabla 1. Aporte energético (\%) de cada ingrediente en las raciones y consumo total aves

\begin{tabular}{cccc}
\hline Tratamiento & Dieta & $\begin{array}{c}\text { Clase de } \\
\text { consumo }\end{array}$ & $\begin{array}{c}\text { Consumo } \\
\text { Total }(\mathbf{g})\end{array}$ \\
\hline 1 & Alimento balanceado comercial & Voluntario & 5072 \\
2 & TSF $+80 \%$ APA $+20 \% \mathrm{HA}^{*}$ & Restringido & 3550 \\
3 & $\mathrm{TSF}+(90 \% \mathrm{APA}+10 \% \mathrm{AS})+20 \% \mathrm{HA}^{*}$ & Restringido & 3550 \\
4 & $\mathrm{TSF}+(80 \% \mathrm{APA}+20 \% \mathrm{AS})+20 \% \mathrm{HA}^{*}$ & Restringido & 3550 \\
\hline
\end{tabular}

*Los valores fueron estimados tomando 4,4 megacalorias $/ \mathrm{kg}$ de dieta, que es el contenido energético de T2, T3 y T4. TSF: Torta de soya fortificada; APA: Aceite de palma africana; HA: Harina de arroz y AS: Aceite de seje o Unama

\section{Extracción del aceite de Seje}

La extracción de aceite utilizado en la dietas para pollo de engorde, se detalla en la Figura 1, donde se logró un manejo adecuado de temperatura durante todo el proceso, utilizando de una prensa de tornillo sin fin para obtener un mayor rendimiento de la cantidad de aceite. 
Tabla 2. Mezcla de los alimentos utilizados como base nutricional para los tratamientos suministrados a pollos de engorde

\begin{tabular}{lcccc}
\hline \multicolumn{1}{c}{ Producto (\%) } & T1 & T2 & T3 & T4 \\
\hline Concentrado comercial & 100 & - & - & - \\
Torta de soya & - & 50 & 50 & 50 \\
Harina de arroz & - & 9.5 & 9.5 & 9.5 \\
Tricalfos & - & 0.6 & 0.6 & 0.6 \\
Vitaminas & - & 0.3 & 0.3 & 0.3 \\
Minerales & - & 0.3 & 0.3 & 0.3 \\
Sal & - & 0.3 & 0.3 & 0.3 \\
Aceite de palma africana & - & 39 & 35 & 31 \\
Aceite de seje Total & - & - & 4 & 8 \\
& $\mathbf{1 0 0}$ & $\mathbf{1 0 0}$ & $\mathbf{1 0 0}$ & $\mathbf{1 0 0}$ \\
Energía metabólica Mcal/kg de alimento & 3,2 & 4,4 & 4,4 & 4,4 \\
Proteína (\%) & 22 & 21,9 & 21,9 & 21,9 \\
\hline
\end{tabular}

\section{Fruto Extraído

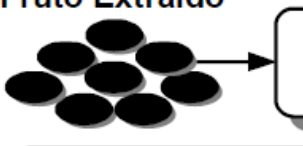 \\ Calentamiento del Fruto \\ $t^{\mathrm{a}}$ Cercana a los $40^{\mathrm{a}} \mathrm{C}$ \\ Cocción de Residuo de (Cáscara y Mesocarpio) en Caldera de Cobre con ta aprox. de $70^{\mathrm{a}} \mathrm{C}$}

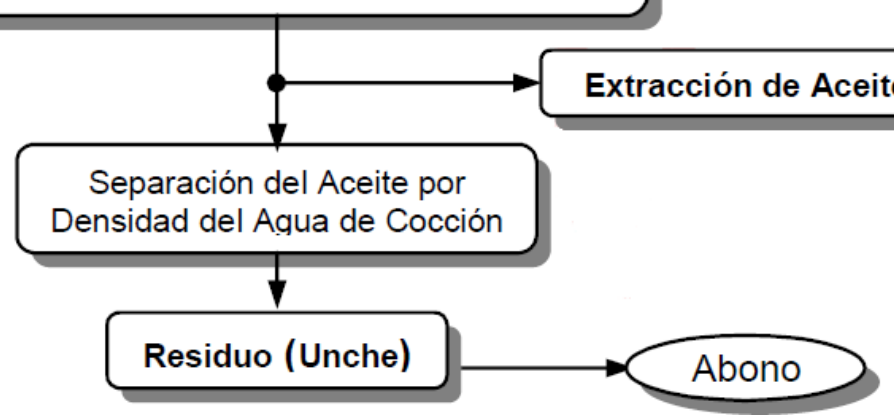

Figura 1. Procedimiento para la extracción del aceite de Seje. Tomado de Ocampo et al., (2013)

\section{RESULTADOS Y DISCUSIÓN}

En la primera y segunda semana el aumento de peso fue similar para los cuatro tratamientos $(P<0.420$ y $P<0.307$, respectivamente), mientras que en la tercera el incremento fue mayor $(\mathrm{P}<0.035)$ para $\mathrm{T} 1$ y $\mathrm{T}$, observándose igual comportamiento entre sí (Tabla 3 y Figura 2). La cuarta y quinta semana T2 fue el de menor aumento $(P<0.047$ y $P<0.083)$, no se presentaron diferencias entre $T 1$, 
T3 y T4. La ganancia de peso día/ave fue poco significativa $(P>0.082)$, demostrando una ganancia de peso similar entre los tratamientos, el porcentaje de rendimiento en canal fue superior (0.010) para T1 seguido en su orden por T3 y T4 (10 y $20 \%$ de AS, respectivamente) y por ultimo T2, (0\% de AS) (Tabla 3 ).

Tabla 3. Promedios de variables evaluadas por tratamiento

\begin{tabular}{|c|c|c|c|c|c|}
\hline Variable & T1 & T2 & T3 & T4 & $\mathbf{P}$ \\
\hline $\begin{array}{l}\text { Peso vivo promedio inicial } \\
\text { ave }(\mathrm{g})\end{array}$ & 69.65 & 69.65 & 69.65 & 69.65 & \\
\hline Numero de aves inicial & 19 & 19 & 19 & 19 & \\
\hline $\begin{array}{l}\text { Peso vivo promedio inicial } \\
\text { Tto/ave }(\mathrm{g})\end{array}$ & $326 \pm 19$ & $322 \pm 15$ & $305.8 \pm 18$ & $348.6 \pm 23$ & \\
\hline $\begin{array}{l}\text { Peso semana } 1 \text { Tto/ave (g) } \\
\text { (acostumbramiento) }\end{array}$ & $509.6 \pm 9$ & $497.5 \pm 40$ & $547.5 \pm 40$ & $552.6 \pm 80$ & 0.420 \\
\hline Peso semana 2 Tto/ave (g) & $813.3 \pm 19$ & $769.5 \pm 40$ & $841.3 \pm 50$ & $857.3 \pm 100$ & 0.307 \\
\hline Peso semana 3 Tto/ave $(\mathrm{g})$ & $1012 \pm 30^{\mathrm{a}}$ & $955.3 \pm 65^{a}$ & $1136 \pm 115^{b}$ & $1189.6 \pm 73^{b}$ & $0.035^{\star}$ \\
\hline Peso semana 4 Tto/ave $(\mathrm{g})$ & $1394.3 \pm 120^{b}$ & $1216.6 \pm 45^{a}$ & $1496.3 \pm 100^{b}$ & $1390.3 \pm 120^{b}$ & $0.047^{*}$ \\
\hline Peso semana 5 Tto/ave $(\mathrm{g})$ & $1688 \pm 150$ & $1501 \pm 20$ & $1836.6 \pm 130$ & $1678.6 \pm 130$ & 0.083 \\
\hline $\begin{array}{l}\text { Ganancia de peso total } \\
\text { (g/ave) }\end{array}$ & 1362 & 1179 & 1530.8 & 1678.6 & 0.082 \\
\hline Consumo total alimento/ave & 5072 & 3550 & 3550 & 3550 & \\
\hline Conversión alimenticia & $3.72^{\mathrm{a}}$ & $3.01^{\mathrm{b}}$ & $2.31^{\mathrm{c}}$ & $2.51^{\mathrm{b}}$ & $0.001^{\star *}$ \\
\hline$\%$ Rendimiento en canal & $66.67 \pm 2^{a}$ & $58.2 \pm 3^{b}$ & $\begin{array}{l}61.87 \\
\pm 0.5^{c}\end{array}$ & $63.25 \pm 3^{c}$ & $0.010^{* \star}$ \\
\hline
\end{tabular}

Letras diferentes en la fila indica diferencia estadística. ${ }^{\star}$ Es significativo, ${ }^{\star \star}$ Es altamente significativo

Estos resultados indican que el uso de ácidos grasos en gran proporción (saturados : poliinsaturados) del APA y AS en la dieta, pueden sustituir eficientemente el uso de carbohidratos (Figura 2), igualando o mejorando el sistema metabólico para obtener energía (ATP) manteniéndolo positivo, esto se puede justificar, por las reacciones bioquímicas en el Ciclo de Krebs, donde la mayoría de los compuestos enzimáticos están constituidos por vitaminas del complejo B, cuando se utilizan dietas altas en grasas, se ha comprobado en aves, que se ahorran estas vitaminas, reduciendo el calor producido en las reacciones metabólicas, y por tanto el estrés térmico (Macari et al., 2004). También ha sido comprobado en la especie porcina por Ocampo, (2004) y Terán, (2003) cuando energía digestible fue suplida por lípidos (aceites) se incrementó el peso por animal en comparación con dietas comerciales que tienen un aporte energético a base de carbohidratos (granos de cereales) confirmando estudios anteriores 
realizados por Herrera y Quintero, (1996) Jorgensen, (2000) de la Llata et al., (2001) y Ocampo, (2002).

La conversión alimenticia menos eficiente fue para $T 1(\mathrm{P}<0.001)$, donde el mejor resultado lo obtuvo T3, que requirió $2.31 \mathrm{~g}$ de alimento para aumentar un gramo de peso, comportamiento similar se observó en T2, superando ambos a T1. Por otro lado, el porcentaje de rendimiento en canal fue mayor $(P<0.01)$ para los tratamientos tres $(61.9 \%)$ y cuatro $(63.3 \%)$, en los que no se observaron diferencias entre sí, pero si con los T1 (66.7\%) y T2 (58.2\%) (Tabla 3); aunque todos los tratamientos estuvieron por debajo del rendimiento esperado en la raza Cobb que es $77.66 \%$ (Cobb-vantress, 2013), con estos valores se puede evidenciar que las condiciones ambientales afectaron el consumo y su eficiencia para producción de carne, además se observó que las aves respondieron al consumo de ácidos grasos en dietas restringidas, de forma similar a los pollos alimentados con concentrados basados en carbohidratos con alimento no restringido.

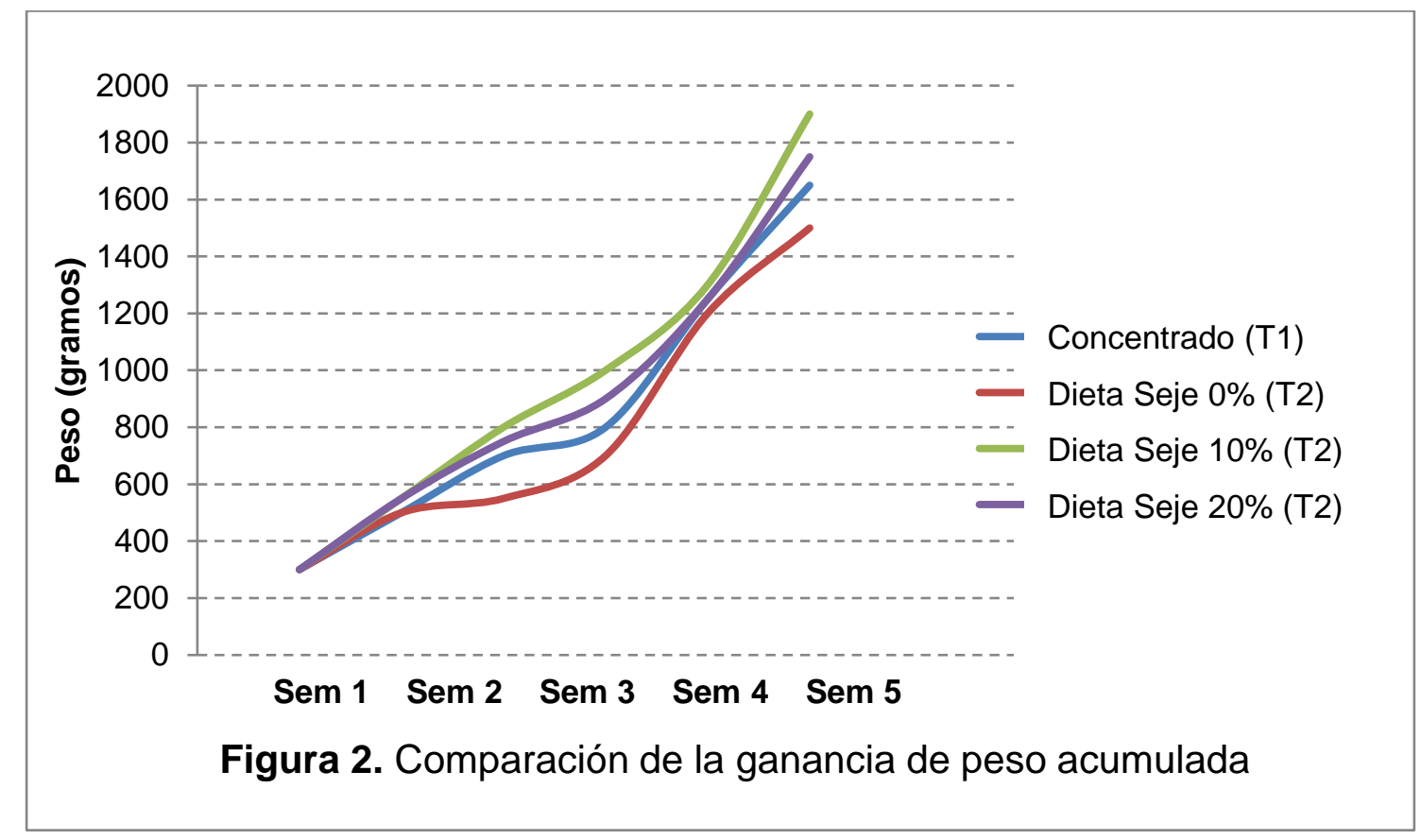

La causa del menor rendimiento de T2 con solo aceite crudo de palma africana (APA) en comparación con T3 y T4, que tenían incluido no solo APA sino aceite de 
seje (AS), posiblemente se debió porque el APA contiene elevados niveles de ácido oleico y palmítico, que son fuentes de ácidos grasos monoinsaturados (AGM), aunque tienen una respuesta sinérgica en la alimentación, se observó un efecto aditivo del AS, que contiene además, otros lípidos polinsaturados como ácido linoleico y linolénico), los cuales pueden estar en una proporción que se refleja en un mayor peso y eficiencia alimenticia en el animal (Ocampo, 2002; Rodríguez et al., 2002).

El uso de lípidos en las dietas se puede considerar con un valor energético 3.8 veces superior al de los carbohidratos como fuente de energía (Belitz et al., 1998); por lo cual el T1 obtuvo una menor conversión alimenticia comparada con la de pollos de engorde alimentados con aceites, esto indica mayor eficiencia de los lípidos con relación a los carbohidratos cuando se suministran, dietas a aves en condiciones de clima cálido (Jahan et al., 2005), además el T1 no logro una conversión alimenticia similar o igual a la presentada por la incubadoras en Colombia (1.24) (Cobb-vantress, 2013), lo cual se tiene como referencia en el periodo de engorde, cuando las aves son alimentadas con dietas comerciales a base de granos.

El porcentaje de mortalidad fue $2.5 \%$ del total de aves, el experimento se inició con 80 aves pero en el momento de las adaptaciones al consumo del aceite murieron dos aves, por lo tanto se sacaron seis aves, dejando 72 para establecer igualdad numérico en las repeticiones. El problema de mortalidad fue causado por una enteritis producida posiblemente por bacterias Escherichia coli y Salmonella spp. La colibacilosis en aves es un problema secundario ligado a contaminación de la fuente de agua con materia fecal (Carranza et al., 2012), además en el periodo seco aumenta la prevalencia de bacterias oportunistas (Rubio et al., 2010), lo cual pudo desencadenar lesiones observadas como artritis, enteritis y sinovitis; así mismo Salmonella puede ser también oportunista en casos de colibacilosis (Bustos y Segura, 2005), este cuadro clínico cursó con diarrea sanguinolenta presentada en los animales bajos los tratamientos uno y dos; el problema se resolvió con la administración de protectores de mucosa intestinal y 
aumento a 50 ppm de cloro por litro de agua para todas las aves. Por otro lado, los tratamientos tres y cuatro presentaron signos muy leves de la contaminación por E. coli como heces blandas sin sangre; esto puede indicar que la adición del aceite de seje y el uso de vitamina $A$, posiblemente incrementaron la inmunidad de las aves, reparando las células del epitelio de las mucosas, mejorando la actividad del sistema inmune del tracto digestivo, además de estimular la actividad de fagocitos y linfocitos (Edem, 2002).

\section{Observaciones de la canal}

El sacrificio de las aves se realizó por medio del desangrado, se continuó con el desplumado y eviscerado de las mismas; en T1 se observó una grasa pegajosa amarilla, de una consistencia muy firme, no esponjosa, muy adherida a la canal y se encuentra en gran cantidad sobre la región abdominal y del costillar, además el marmóreo encontrado en las aves de este tratamiento fue menor en comparación a las canales de los otros tratamientos.

En T2, T3 y T4 las canales presentaron una consistencia de grasa similar blanda y esponjosa de color blanco, en T2 y T3, los lípidos estuvieron poco adheridos en comparación al T4 que mostró mayor fijación al músculo, siendo su distribución uniforme dentro de sus fibras, observándose de tres a cuatro líneas de grasa dentro de los músculos pectorales y abdominales, por lo cual estas canales presentaron buen marmóreo (Tabla 4).

Tabla 4. Descripción de la grasa abdominal de las canales de los pollos de engorde

\begin{tabular}{ccccc}
\hline Observación & T1 & T2 & T3 & T4 \\
\hline Cloro grasa & Amarilla & Blanca & Blanca & Blanca \\
Consistencia & Dura & Blanda & Semi blanda & Semi blanda \\
Adherencia & Alta & Baja & Baja & Media \\
Marmóreo & Poco visible & Muy visible & Muy visible & Muy visible \\
Color carne & Amarillo claro & Rosado claro & Rosado claro & Rosado claro \\
\hline Existe una relación entre el color y la consistencia de la grasa muscular, cuando \\
\multicolumn{4}{c}{ contiene altos niveles de ácidos } & grasos insaturados es blanca y con mayor
\end{tabular}


proporción de ácidos grasos saturados es amarilla. La cantidad aportada de aceites y grasas en la dieta ayudan a mejorar el sabor, color y firmeza de la carne (Ospina et al., 2011; Jahan et al., 2005). Además la consistencia de la grasa tiene una gran importancia porque determina la apariencia y facilidad de manipulación de la canal, y ésta depende fundamentalmente de la proporción de triglicéridos que se encuentran en forma líquida o sólida a una determinada temperatura, es decir que a mayor número de enlaces instaurados en las cadenas de ácidos grasos que constituyen los triglicéridos de la canal, es mejor en comparación cuando la grasa es sólida en la cobertura de la piel, la intermuscular e intramuscular, afectando la consistencia del marmóreo (López, 2004). Suministrar ácidos grasos polinsaturados produce canales con mejor marmóreo en comparación con animales que son alimentados con grasas saturadas, que posiblemente eran las fuente de lípidos en el concentrado (Pérez, 2011; Nelson et al., 2004; Jahan et al., 2005).

\section{Degustación carne de pollo}

Las degustaciones se realizaron inicialmente en el grupo familiar de la Reserva Las Unamas, seguido del grupo de trabajadores y grupo familiar de técnicos colaboradores; los diferentes grupos que degustaron la carne de pollo lo hicieron sin saber a qué tratamiento pertenecían las aves a aprobar, la carne se preparó asada para no agregar condimentos que alteraran su sabor, cada degustador probó la misma presa de las aves de los diferentes tratamientos y se les pidió que catalogaran a gusto personal, cuál era la carne que por su sabor, olor y textura les agradó más para consumo, la carne de los tratamientos se les suministró a un total de 30 personas para su degustación (Tabla 5).

El 43.3\% (13 personas) del grupo de degustadores escogieron como la carne de primera preferencia por su olor, sabor y textura al T3 (90\% APA y $10 \%$ AS), la segunda preferencia fue para el T4 (80\% APA y $20 \%$ AS) con un $50 \%$ de preferencia. La tercera preferencia fue para el T2 (100\% APA) con el $33.3 \%$, y la cuarta y última preferencia fue para la carne del T1 (alimento balanceado comercial) con el $0 \%$ de elección por parte de los degustadores (Tabla 5). 
Tabla 5. Análisis general de los grupos de degustación

\begin{tabular}{lcccccccc}
\hline \multirow{2}{*}{ Tratamiento } & \multicolumn{2}{c}{ Preferencia T1 } & \multicolumn{2}{c}{ Preferencia T2 } & \multicolumn{2}{c}{ Preferencia T3 } & \multicolumn{2}{c}{ Preferencia T4 } \\
& Personas & $\%$ & Personas & $\%$ & Nersonas & $\%$ & Personas & $\%$ \\
\hline T1 Concentrado & - & 0 & 3 & 10 & 6 & 20 & 21 & 70 \\
T2 100\% ACP & 8 & 26.7 & 7 & 23.3 & 10 & 33.3 & 4 & 13.3 \\
T3 10\% AS & 13 & 43.3 & 5 & 16.7 & 8 & 26.7 & 4 & 13.3 \\
T4 20\% AS & 9 & 30 & 15 & 50 & 6 & 20 & 1 & 13.3 \\
\hline
\end{tabular}

\section{Costos de dietas experimentales}

El costo de las dietas (Tabla 6) que contenían como fuente de energía ácidos grasos provenientes de aceites, fue menor en comparación con la dieta de cereales, la diferencia fue una disminución del 30\% de los gastos de alimentación, lo cual reduce los costos totales de producción.

Tabla 6. Comparación de costos de las diferentes dietas suministradas en pollos de engorde

\begin{tabular}{lcccc}
\hline \multicolumn{1}{c}{ Materias Primas } & T1 CC & T2 APA & T3 10\% AS & T4 20\% AS \\
\hline Consumo total alimento / ave (g) & 5.072 & 3.550 & 3.550 & 3.550 \\
Torta de soya (\$) & & 1.352 & 1.352 & 1.352 \\
Harina de arroz (\$) & & 202 & 202 & 202 \\
Tricalfos (\$) & & 70 & 70 & 70 \\
Sal $(\$)$ & & 15 & 15 & 15 \\
Vitaminas (\$) & & 206 & 306 & 306 \\
Minerales (\$) & & 1.180 & 1.059 & 275 \\
Aceite de palma africana (\$) & & - & 93 & 186 \\
Aceite de seje (\$) & 4.690 & 3.400 & 3.372 & 3.344 \\
Valor dieta / ave (\$) & 89.110 & 64.609 & 64.100 & 63.536 \\
Valor Total Tratamiento (\$)
\end{tabular}

${ }^{*} \mathrm{CC}=$ Concentrado comercial, APA = Aceite de palma africana, $\mathrm{AS}=$ Aceite de seje

El menor costo fue para los tratamientos que manejaron la inclusión de aceite de seje: $80 \%$ APA y $20 \%$ AS (T4) y en un $90 \%$ APA y $10 \%$ AS (T3), esto demuestra que con la inclusión de ácidos grasos en la dieta para pollos de engorde, se mejora la relación de ácidos polinsaturados : saturados, se logra una mayor o igual eficiencia en comparación a las dietas suministradas tradicionalmente en la producción animal a base de cereales. Igualmente la adición de aceites en las dietas para pollos de engorde brinda un menor consumo de alimento 
disminuyendo su costo y con una aceptación mayor en la calidad del producto final para el consumidor.

\section{CONCLUSIONES}

Es viable el uso de dietas ricas en ácidos grasos para la alimentación de pollos de engorde, aunque la ganancia de peso fue similar para todos los tratamientos (cereal vs aceites), pero en términos de conversión alimenticia fue mejor en las dietas ricas en ácidos grasos (aceites). Por otro lado, el tratamiento uno a base de concentrado comercial presento mayor rendimiento en canal seguido de los tratamientos que se les incluyó aceite de seje. La carne de pollo producida con dietas ricas en grasa fue preferida en comparación con el tratamiento uno.

Aparentemente la inclusión en el total de la dieta del aceite de seje (10 y 20\%) adicionando aceite de palma africana como fuente energética, afectaron positivamente la composición final de ácidos grasos de la carne de pollo favoreciendo su consistencia y palatabilidad.

\section{REFERENCIAS BIBLIOGRÁFICAS}

1. Alfonso N. Potencial de aprovechamiento de las palmas proveedoras de frutos alimenticios de la Orinoquia Colombiana. Trabajo de grado Bióloga, Facultad de Ciencias, Pontificia Universidad Javeriana, Bogotá, Colombia 49 p. 2009.

2. Aparicio I. Aprovechamiento de subproductos de leguminosas para la obtención de productos funcionales. comparación de metodologías para la caracterización de la fibra alimentaria. Tesis de Grado Doctoral, Facultad de Farmacia, Departamento de Nutrición y Bromatología, Universidad Complutense de Madrid. 235 p. 2008.

3. Avila H. Comparación de las ventajas de desodorización en una refinería física "Multistock" respecto a una refinería física "unistock". Trabajo de Grado Ingeniero Químico, Facultad de Ingeniería, Universidad de San Carlos de Guatemala, 59 p. 2006.

4. Balick MJ. Jessenia y Oenocarpus: Palmas aceiteras neotropicales dignas de ser domesticadas. Estudio FAO Producción y protección vegetal 88. Roma FAO. 1992.

5. Banco de Occidente. La Orinoquia de Colombia. I/M Editores. Cali, Colombia. 2005. Disponible En: http://www.imeditores.com/banocc/orinoquia/presentacion.htm

6. Belitz HD, Grosch W, Schielberle P. Lipids. En: Food Chemistry. $3^{\mathrm{a}}$ Ed. Berlín, Springer: p 157-244. 2004.

7. Bustos P, Segura C. Incidencia de Salmonella (Salmonella ssp) y E. coli en tres granjas porcícolas ubicadas en los municipios de Fómeque y Sibaté. Trabajo de Grado Zootecnista. Facultad de Zootecnia, Universidad de la Salle, Bogotá. 85 p. 2005. 
8. Borchsenius F, Moraes M. Diversidad y usos de palmeras andinas (Arecaceae). Botánica Económica de los Andes Centrales, p 412-433. 2006.

9. Coromoto M. Usos y efectos de la incorporación de grasas y aceites en dietas para cerdos. Universidad central de Venezuela. Journal of Animal Science, 87 (14): 15461560. 2004.

10. Carranza C, Léon R, Falcón N, Neumann A, Kromm C. Caracterización y distribución de cepas de Escherichia coli potencialmente patógenos aisladas de pollos broiler de explotaciones avícolas en el Perú. Rev Inv Vet Perú, 23 (2): 209-219. 2012.

11. Cobb-vantress Inc. Cobb Guía de manejo del pollo de engorde. 63 p. 2013. Disponible En: http://www.cobb-vantress.com/docs/default-source/guides/cobbbroiler-management-guide---spanish.pdf

12. Correa, H. D, Ruiz, S. L., Arévalo, L. M. (ed.) Plan de acción en biodiversidad de la cuenca del Orinoco - Colombia / 2005 - 2015 - Propuesta Técnica. Bogotá D.C.: Corporinoquia, Cormacarena, I.A.v.H, Unitrópico, Fundación Omacha, Fundación Horizonte Verde, Universidad Javeriana, Unillanos, WWF - Colombia, GTZ Colombia. 273 p. 2005.

13. De la Llata M, Dritzz S, Tokach MD, Goodband RD, Nelssen JL, Loughin TM. Effects of dietary fat on growth performance and carcass characteristics of growing-finishing pigs reared in a commercial environment. Journal of Animal Science, 79 (10): 26432650. 2001.

14. Delgado C, Rosegrant M, Steinfeld H, Ehui S, Courbois C. Livestock to 2020. The next food revolution. Food, Agriculture and the Environment discussion paper N. 28, Washington DC, International Food Policy Research Institute, Rome, FAO, and Nairobi, International Livestock Research Institute. 1999.

15. Edem D. Palm oil: biochemical, physiological, nutritional, hematological and toxicological aspects: a review. Plant Foods Hum Nutr Fall, 57 (3-4): 319-341. 2002.

16. Herrera A, Quintero W. Utilización del aceite de crudo de Palma Africana como fuente de energía en la alimentación de pollos de engorde en semiconfinamiento durante la fase de finalización. Tesis de pregrado Médico Veterinario Zootecnista, Universidad de los Llanos, Villavicencio, Colombia. 1996.

17. Instituto de Hidrología, Meteorología y Estudios Ambientales (IDEAM). Información Histórica, Climatografía de las principales ciudades, Cartas Climatológicas - Medias Mensuales, $2014 . \quad$ Disponible En: http://atlas.ideam.gov.co/visorAtlasClimatologico.html

18. Jahan K, Paterson A, Piggott J, Spickett C. Chemometric modeling to relate antioxidants, neutral lipid fatty acids and flavor components in chicken breasts. Poultry Science, 84 (1): 158-166. 2005.

19. Jorgensen $H$, Gabert VM, Hedemann M, Jensen S. Digestion of fat does not differ in growing ping fed diets containing fish oil, rapeseed oil or coconut oil. Journal of Nutrition, 130 (4): 852-857. 2000.

20. López C. Control, mediante la alimentación animal, de la composición de la carne. Facultad de Veterinaria, Universidad Complutense. España. 2002. Disponible En: http://racve.es/publicaciones/control-mediante-la-alimentacion-animal-de-lacomposicion-de-la-carne/

21. Macari M, Furlan RL, Maiorka A. Aspectos fisiológicos e de manejo para manutenção da homeostase térmica e controle de síndromes metabólicas. En: Mendes AA, Naas IA, Macari M. Produção de frangos de corte. Campinas: Facta, p 137-156. 2004. Disponibles En: http://www.elsitioavicola.com/articles/2676/estras-calarico-en-laproduccian-de-pollos-3-alimentacian/\#sthash.upidflGq.dpuf 
22. Merinova EM, Seizova KA, Totseva IR, Panayotova S, Marekov I, Momchilova S. Oxidative changes in some vegetable oils during heating at frying temperature, Bulgarian Chemical Communications, 44 (1): 57-63. 2012.

23. Nelson M, Marks D, Busboom J, Cronrath J, Falen L. Effects of supplemental fat on growth performance and quality of beef from steers fed barley-potato product finishing diets: I. Feedlot performance, carcass traits, appearance, water binding, retail store, and palatability attributes 1. Journal of Animal Science, 82 (12): 3600-3610. 2004.

24. Ocampo A. High lipid diets based on palm oil for growing-fattening pigs. Thesis Doctoral, University of London. Imperial College (Wye Campus). Department of Agricultural Sciences. 352 p. 2002.

25. Ocampo A, Fernández A, Castro F. Aceite de palma de seje Oenocarpus bataua Mart. por su calidad nutricional puede contribuir a la conservación y uso sostenible de los bosques de galería en la Orinoquia Colombiana. Rev. Orinoquia, 17 (2): 215-229. 2013.

26. Ordoñez I. Elaboración de suplementos nutricionales con base en el uso integral de las plantas de yucca (Manihot esculenta Crantz) y batata (Ipomea batatas Lam), por medio de extrusión para la alimentación de monogástricos. Tesis de grado, Facultad de Ingenierías, Universidad de San Buenaventura, Santiago de Cali. 115 p. 2006.

27. Ocampo A. Cambio del patrón de energía para producir carne: las dietas ricas en grasa basadas en aceite de palma, una oportunidad para los países tropicales. Rev Palmas, 25 (1): 275-287. 2004.

28. Ospina S, Restrepo D, López J. Derivados cárnicos como alimentos funcionales. Revista Lasallista de Investigación, 8 (2): 163-172. 2011.

29. Peñuela L, Castro F, Ocampo N. Las reservas naturales del Nodo Orinoquia en su rol de conservación de la biodiversidad. Fundación Horizonte Verde y Resnatur. Colombia. 104 p. 2011.

30. Pérez B. Suplementaciones de raciones para cebo intensivo de ternero con aceites vegetales: rendimientos productivos, calidad de la canal, de la grasa y de la carne. Tesis de Grado Doctoral. Facultad de Veterinaria, Departamento de Producción Animal, Universidad Complutense de Madrid, España. 317 p. 2011.

31. Rodríguez ML, Ortíz L, Alzueta C, Rebolé C, Treviño J. Nutritive value of high-oleic acid sunflower seed for broiler chickens. Poultry science, 84 (3): 395-402. 2005.

32. Rossell, JB. Chemical changes in soy oil during high temperature processing. En: Proceedings of the second ASA Symposium on Soybean Processing, Leysen RJ (Ed), American Soybean Association, Brussels, Belgium. 1981.

33. Rubio M, Alcides C, Silveira R, Aguilera Y. Variabilidad bacteriana en Oreochromis sp durante las estaciones de lluvia y seca, cultivadas en ambiente dulceacuícola en diferentes regiones de Cuba. REDVET. Revista Electrónica de Veterinaria, 11 (7): 1 11. 2010.

34. Segers JC, Van de Sande R. Degumming theory and practice. Proceedings AOCS World Conference on Edible Fats and Oils Processing, Maastricht: p 83-88. 1988.

35. Terán G. Uso del aceite crudo de Palma (Elaeis guineensis) como fuente energética en dietas para cerdos en crecimiento y finalización. Tesis Maestría en Producción Animal Tropical, Facultad de Medicina Veterinaria y Zootecnia, Universidad Autónoma de Yucatán, México. 2003.

36. Yaulema D. Regeneración de aceites comestibles. Tesis de Pregrado Ingeniero Químico. Facultad de Ingeniería Química, Universidad de Guayaquil. Ecuador. 80 p. 2014.

37. Žilić S, Šobajić S, Mladenović S, Mladenović B, Vasić M. Effects of heat processing on soya bean fatty acids content and the lipoxygenase activity. Journal of Agricultural Sciences, 55 (1): 55-64. 2010. 